\title{
A Learning Analytics Approach to Correlate the Academic Achievements of Students with Interaction Data from an Educational Simulator
}

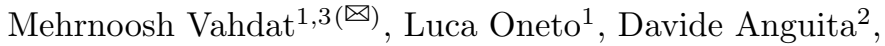 \\ Mathias Funk ${ }^{3}$, and Matthias Rauterberg ${ }^{3}$ \\ 1 DITEN - Università degli Studi di Genova, 16145 Genoa, Italy \\ mehrnoosh.vahdat@edu.unige.it, luca.oneto@unige.it \\ 2 DIBRIS - Università degli Studi di Genova, 16145 Genoa, Italy \\ davide.anguita@unige.it \\ 3 Department of Industrial Design, Eindhoven University of Technology, \\ 5612AZ Eindhoven, The Netherlands \\ \{m.funk,g.w.m.rauterberg\}@tue.nl
}

\begin{abstract}
This paper presents a Learning Analytics approach for understanding the learning behavior of students while interacting with Technology Enhanced Learning tools. In this work we show that it is possible to gain insight into the learning processes of students from their interaction data. We base our study on data collected through six laboratory sessions where first-year students of Computer Engineering at the University of Genoa were using a digital electronics simulator. We exploit Process Mining methods to investigate and compare the learning processes of students. For this purpose, we measure the understandability of their process models through a complexity metric. Then we compare the various clusters of students based on their academic achievements. The results show that the measured complexity has positive correlation with the final grades of students and negative correlation with the difficulty of the laboratory sessions. Consequently, complexity of process models can be used as an indicator of variations of student learning paths.
\end{abstract}

Keywords: Learning analytics $\cdot$ Educational data mining $\cdot$ Technology Enhanced Learning - Process mining - Complexity • Interaction data • Educational simulator

\section{Introduction}

Learning Analytics (LA) and Educational Data Mining (EDM) have raised a lot of attention among researchers and practitioners of Technology Enhanced Learning (TEL) in the last decade. The aim is to gain more insight into the behavior of learners by building models based on data collected from learning

(C) Springer International Publishing Switzerland 2015

G. Conole et al. (Eds.): EC-TEL 2015, LNCS 9307, pp. 352-366, 2015.

DOI: $10.1007 / 978-3-319-24258-3 \_26$ 
tools [1]. Consequently LA and EDM applied in educational settings enhance the understanding of various stakeholders (students, teachers, administrators, etc.) about the way people learn in a data-driven way. As a result, the TEL systems can be improved to be more personalized and adaptive, and learning can be optimized as one of the main goals [2-5].

In the environments where inquiry-guided learning is applied rather than traditional methods, e-tools can play an important role in providing guidance and principles to optimize learning. Inquiry-guided learning focuses on contexts where learners are meant to discover knowledge rather than passively memorizing the concepts [6-8]. In this way, the lesson begins with a set of observations to interpret, and the learner tries to analyze the data or solve the problem by the help of the guiding principles [9], resulting in a more effective educational approach [10]. In this context, simulation-based environments are efficient tools to improve learning [11]. The data gathered from these educational environments has special features of student-performed actions $[12,13]$. Therefore, to analyze this data we need to integrate methods that focus on learning processes in a more effective way. Among process related methods, Process Mining (PM) is emerging in TEL since it can enhance our understanding by building models of student actions and learning processes [14].

This paper is structured as follows. In Sect. 2, PM and its applications in education are described. We introduce a metric for measuring the understandability of process models, and the main contribution of this study is presented. In Sect. 3, PM tools and CM metric are described. In Sect.4, we describe the protocol and how we collect the data. In Sect. 5, we report the results of this study and finally in Sect.6, conclusions of this study are drawn.

\section{Related Work and Contribution}

\subsection{Educational Process Mining}

Process Mining is a method that has emerged from the business community to obtain valuable knowledge from a process. By adopting various PM techniques such as process model discovery and conformance checking, processrelated knowledge from event logs can be extracted. In the context of LA and EDM, very few process-oriented approaches focus on the process as a whole [14]. Contrarily, educational PM allows obtaining a better insight on the underlying educational processes [15]. For example in [16], some applications of PM methods are shown and applied to online assessment data for analyzing the process of answering the questions or requesting feedback by students. In [15], a framework is introduced for integrating the domain knowledge in educational PM in order to facilitate interactive PM and help educators analyze educational processes based on formal modeling. Another study [17] analyzes student processes of self-regulated learning and shows the differences of learning behavior between successful students and less successful ones from their temporal patterns. For instance, it shows that successful students perform regulatory activities with a higher frequency and in a different order than the less successful ones. 


\subsection{Understanding Process Mining Through a Complexity Metric}

In PM, many difficulties are on the way of understandability of process models. In [18] it is shown that, although process models help people have better understanding of processes, understanding complex models faces cognitive limits. In this context, complexity metrics can measure understandability and maintainability of a workflow net or a business process model extended from the metrics applied to measure the software complexity $[19,20]$. These metrics in PM have been introduced for petri nets or workflow nets but can be extended to other formalisms as well [21]. For instance, Cyclomatic Complexity of McCabe (CM) has been used as a metric to measure the complexity of a control-flow graph of a program [22], and usually applies to software complexity in the meaning of the difficulty to maintain, change and understand software [23]. CM is measured by calculating the number of linearly-independent paths through a graph [19]. Since complexity has strong relation with various concepts such as simplicity, ease of use, uncertainty and the context of application, it has been a topic of interest to the designers of Human-Computer Interaction (HCI) [24-27]. For instance in [28], this metric is applied into the context of HCI to measure the complexity of the observable behavior by analyzing the recorded task solving process, and obtained the behavioral complexity. In the educational context, CM is used in various studies to measure the complexity of the programming assignments of students while learning [29,30]. For instance, [29] compares CM of children programing tasks when using two interfaces among different age groups. In the next section, we explain how CM is adopted in the context of our study.

\subsection{Cyclomatic Complexity as an Indicator of Student Behavior}

Process Mining is emerging in the field of LA and EDM [15-17]. However, it is challenging to obtain knowledge from educational processes since they are usually very complex and unordered. Metrics like CM have been applied in PM for measuring the understandability of a process model [21]. We further propose to apply CM to understand the complex educational processes and compare them in particular, in the context of inquiry-based learning applications. We apply PM and $\mathrm{CM}$ metric in order to understand the differences between student clusters from their learning paths, while they interact with an educational simulator. For addressing these issues, we carried out our study over a simulation environment named Deeds (Digital Electronics Education and Design Suite) [31,32] which is used for e-learning in digital electronics. The environment provides learning materials through specialized browsers for the students, and asks them to solve various problems with different levels of difficulty. It has been effective, for over ten years, in teaching and improving the learning outcome of students since it provides a general-purpose simulator with a highly interactive e-learning environment. The course is organized in separated theoretical and laboratory sessions where students work with the Deeds simulator. Since we deal with a simulationbased environment, having access to the client side data plays an important role 
in understanding of how the user interacts with the simulator and uses it in problem solving. Therefore, data is gathered through a logging application called LA Data Collector (LADC), properly realized for our purposes. LADC is developed to log data from the client system, and currently can log the interaction data with Deeds browsers and external applications.

This study shows that CM can be used as an indicator of academic success of students. Additionally, it can be used as a predictor of the difficulty level of laboratory sessions for a group of students (e.g. from various engineering majors), and help instructors organize and adapt their lessons based on the needs of the students.

\section{Analytics Methods}

In this study, the data analytics process can be explained in two parts: creating the process models, and comparing them. We used fuzzy miner algorithm which is an effective tool to obtain the process models from the logs of students [17]. Indeed, it improved the abstraction of complex student processes. With this method, we detected the noise (irrelevant activities to the process of learning, for instance, when students setup programs, or visit web pages that are irrelevant to the course) in data and identified the various levels of granularity. Then, we used the obtained knowledge to measure CM effectively, which allowed us to compare the process models of students.

\subsection{Creating the Process Models}

In order to obtain the process models, we used Disco tool, which is developed for discovering models of complex processes through seamless abstraction and generalization [33]. This tool uses the fuzzy miner algorithm, which is effective when the events are unstructured, and the process model is not known beforehand. The fuzzy miner is capable of abstracting and aggregating the process in a way to highlight the most significant parts of the process by measuring the relative importance of behavior (for the formal description of fuzzy miner and its properties refer to [34]). This kind of modeling is required when dealing with so-called spaghetti-like processes since understanding them is very challenging [34]. Figure 1 shows the fuzzy miner capability in aggregating and abstracting a student process model during one of the sessions of the course. On the top, the process is shown with no aggregation, and on the bottom, fuzzy miner presents the most significant and correlated activities and paths by applying the node and edge cutoff parameters [34] to $20 \%$ and $0 \%$ respectively $(0 \%$ is the indicator of minimum number of paths throughout the process). These parameters are set in order to show a simple and yet meaningful process structure.

Applying fuzzy miner for process discovery, facilitated the detection of less significant behavior as well as less related events. By applying this miner to the first level of events, we discovered that out-of-task events (when students are not using the main applications relevant to exercises) are the least significant 


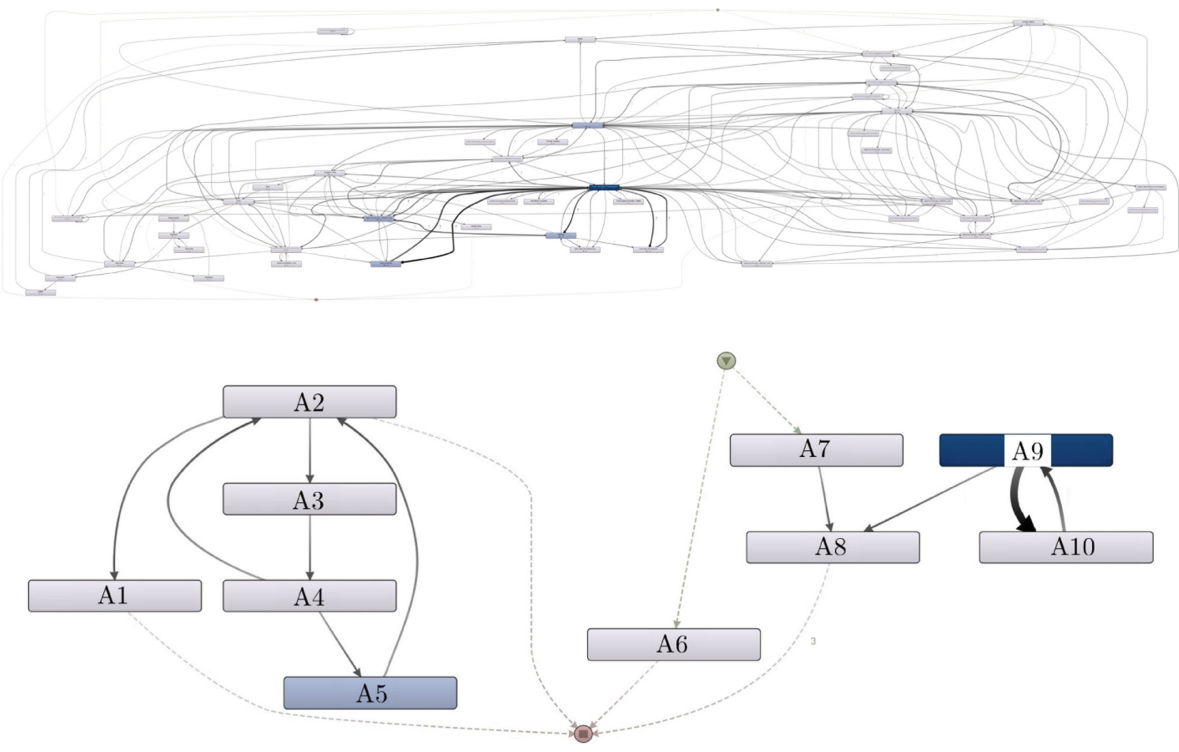

Fig. 1. An example of a student process model obtained by Disco tool and applying the fuzzy miner. Every $A$ refers to an activity (light to dark blue: activities with low to high frequency) (Color figure online).

student behavior in the process of problem solving. In Fig. 2, the process models of a student are shown, considering the $20 \%$ most significant activities. On the top, the process shows the most frequent activities (from light to dark blue) while on the lower image, the most timely activities of student performance are shown (from light to dark red). A comparison of the two showed that there is a lot of noise in data which needs to be reduced by filtering the irrelevant activities. For instance, $A 4$ that is represented with high frequency and low performance time, is an activity that occurs as noise when students switch between the applications.

\subsection{Comparing the Process Models}

In order to compare the process models of students, we measure the complexity of student processes and see if the difference in measured complexity is relevant to the learning achievement. There exist various complexity metrics among which CM [22] is the most widely used metric for measuring the testing efforts of structural programs [20]. CM measures the number of binary decisions (for example IF-statements in a programming language) plus one [19]. This metric is also used to measure the understandability of a process model [21]. In the context of our study, we measure CM of the process models of student interactions, in three levels of granularity, obtained from analysis of the created models. By level of granularity, we mean the way we consider the student performedevents in the context of process models. Events refer to activities and cases in a 

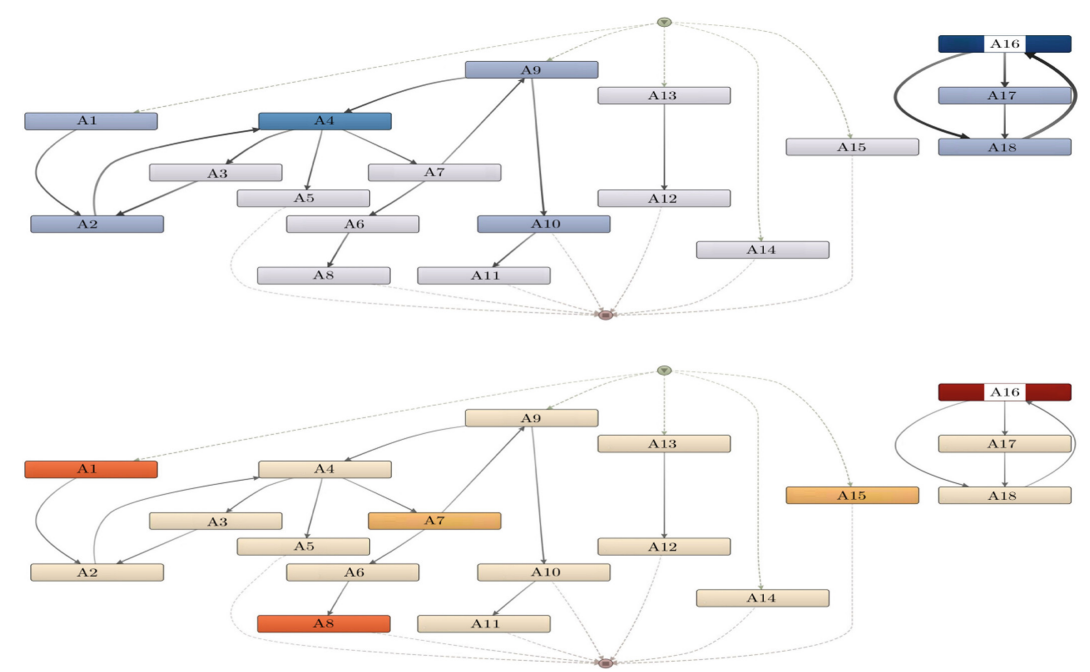

Fig. 2. Comparison of frequency versus performance of a student process model, obtained by Disco tool and applying the fuzzy miner. Every $A$ refers to an activity (on the top, light to dark blue: activities with low to high frequency. On the bottom, light to dark red: activities with low to high performance time) (Color figure online).

workflow process, they are ordered, and obtained from an event log that is recorded sequentially [35]. In the first level, we consider all the events carried out by a student including both in-task and out-of-task events. In the second level, we calculate CM just based on in-task events. During the third level, we group the events based on meta-cognitive activities such that: studying the exercise content, model building, simulating, and reasoning for each specific exercise. Based on [22] for computing CM we need to define $G=(V, E)$ as a control-flow graph where $E$ is the number of independent paths and $V$ is the number of activities. The Cyclomatic metric $C M$ is defined as: $C M=|E|-|V|+P$, where $|x|$ is the cardinality of the set and $P$ is the number of connected components. $P$ in our case is a constant with value of 1 since the process per session is one connected graph with no interruption.

\section{Experimental Design}

This study was carried out on two sets of BSc students during the laboratory sessions of a course of digital design at the University of Genoa. It is performed during the spring semester (March to June 2014) as a pilot experiment, and the autumn semester (October to December 2014). In the framework of this study, 125 students participated in a pilot study and 100 students participated in the real experiment. The structure of the experiment was modified after the pilot to limit the amount of noise in the collected data. For instance, during the pilot, students were free to continue working at home and submit their exercises with 
delay; while in the second round, we set more strict time limits to the process (the result of pilot study are not presented due to the space constraint). Additionally, two people were assigned to help the students during the data collection process in all the sessions. In this way, we ensured the accuracy of data collection by LADC and avoidance of data loss.

A comprehensive experiment has been designed and several activities have been conducted in parallel: semi-structured interviews with the instructors of the course; a demographic questionnaire designed to acquire the general information, motivation, and background knowledge from the learners; and a feedback questionnaire at the end of the course. The designed questionnaire captured the satisfaction of students about the teaching method in the laboratory sessions, working with Deeds simulator, and their feedback on our experiment. In addition, log data were collected from 100 students by LADC from six laboratory sessions. The final exam was designed to address the topics of laboratory sessions separately. In this way, intermediate evaluations of exercises, in addition to the final grade, can be related to the interaction data of each session.
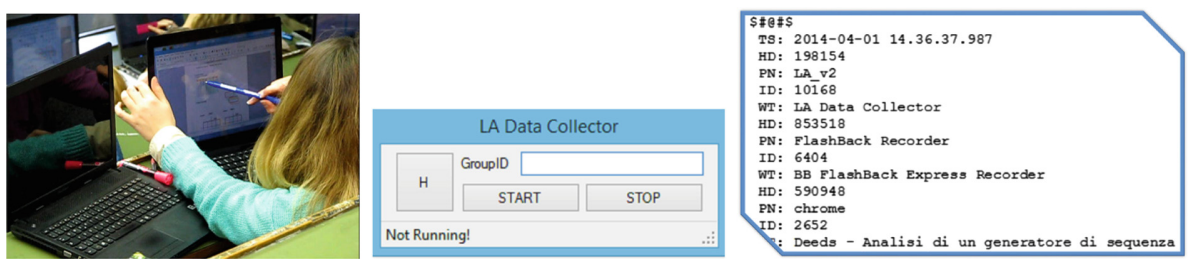

Fig. 3. Process of data collection by LADC in a laboratory session. From left to right: a student performing a task, LADC interface, raw data obtained from LADC.

The process of data collection was performed carefully to maintain the data accuracy and validity. Around $86 \%$ of students in average were present and submitted their logs per session collected by LADC. After each session, testing algorithms were applied on the logs for detecting problems, and in this case, students were informed about their mistakes. In particular, we tested if the logs contained a minimum amount of data, and if data was relevant to the session exercises. Additionally, five students were asked to record their screen in order to have an explicable source for our logs. This data was collected from all the sessions and it was of great help to have more insight on raw data, as well as to understand the reason behind meaningless or irrelevant data in the log.

Figure 3 shows the process of data collection in the classroom by LADC. The raw data was collected as a text file, which contains data of one session per student (we tried to collect as much data as possible without re-engineering the simulator code). This data collects the user interaction every second. Each measure contains data in the following order: date and time, name of application in use on the system in combination with name of its window title, followed by title of the window in focus. Finally data present start and end time of the 
process, idle time, mouse clicks, mouse movements, and keystrokes. The raw data gives us valuable information on the activities per session, per student, and per exercise since each exercise is designed to contain a code in title which appears in the browser, the simulator title, and in the editor used for reasoning. Based on the titles in use, the activities, events and cases are withdrawn sequentially in order to discover the process model of each student through PM. The extracted events are later aggregated into three levels of granularity and CM is measured on each one.

\section{Experimental Results}

The results of this study can be summarized into four main contributions:

- CM is a predictor of difficulty of a session for students, since the average CM per course session has negative correlation with the average of student intermediate grades.

- CM can explain the interactive behavior of students, since the average CM of high-graded students is higher than the one of low-graded students, when calculated per session for two furthest clusters of students.

- CM explains the similarity of student behaviors depending on the level of granularity, and their behavior on out-of-task events.

- CM can raise awareness about the interactive and cognitive behavior of students, and improve the instructional design.

In this paper, we chose to present the results based on the second level of granularity, since from our experiment, this is the best trade-off between the accuracy and compression of the process models. The findings of this study are explained in more depth in the above-mentioned order.

\subsection{Impact of Session Difficulty Over Cyclomatic Complexity}

The results from comparing the average CM of various laboratory sessions, measured from the student processes, show that: CM depends extensively on the difficulty of the assignments given to the students. A comparison of average of intermediate grades $(\overline{I G})$ with average $\mathrm{CM}$ of total students $(\overline{C M})$ is performed. The result shows that $\overline{C M}$ per session has negative correlation with $\overline{I G}$. In Fig. 4, this relationship is shown for the second level of granularity: when $\overline{C M}$ increases, $\overline{I G}$ decreases. A similar result is obtained for the two other levels of granularity. This result can be used for predicting the perceived task difficulty by the students based on their interaction with Deeds simulator. In this way, instructors can spend more time and effort on the topics that are more complicated for the students. Additionally, this course is offered to many engineering majors in University of Genoa, and the students of various intakes have different backgrounds. Therefore, the complexity of the tasks and sessions is not the same for all the classes and majors. In this regard, $\overline{C M}$ shows how difficult or easy a session is for a particular class of students. Later, we compare these results with 
the interviews of the instructors, and show that the findings can be valuable for the teachers in order to organize their lessons and to balance the difficulty of the sessions for the students.

The simulator is new for all students since they are the first year students of computer science engineering. Based on the demographic questionnaire, $74 \%$ of students did not have any experience with the simulator, and the rest had very few experience. So the background knowledge of students about the simulator is considered homogeneous. Additionally, during the first session of the course, complexity of students is relatively high while the average grade is the highest. This is due to the fact that interacting with the system was challenging for them in the beginning, while the assigned exercises were very simple.

\subsection{Impact of Grades over Cyclomatic Complexity}

Another contribution of this study shows the difference of interaction behavior of students when they are clustered into two groups based on their final grades: high-graded versus low-graded students. Our results show that the average of CM of high-graded students is higher than the one of low-graded students. This difference grows when the distance of student clusters increases. We incrementally re-cluster the students by increasing the difference of their grades: high-graded are the one with grades over the mean value of the grades $(\mu)$ plus $n$ times the standard deviation of the grades sigma $(\mu+n \sigma)$ while low-graded students are the ones below $\mu-n \sigma$ where $n$ varies in $\{0,2\}$. Figure 5 shows this difference when $\mathrm{n}$ is 0 (image on the top) versus 2 for the second level of granularity (the lower image). It means when $n=2$ we just consider the most and the least graded students. The results from the second and third level of granularity show a better separation in CM compared to the first level. This is due to the out-of-task events in the first level of granularity which plays as noise in the results. As future directions of this study, we aim to work on exploiting CM to predict the final outcome of each student.
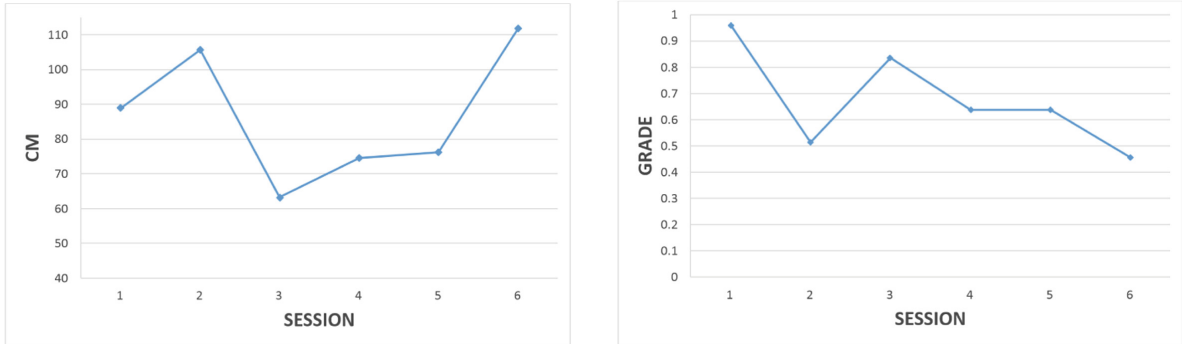

Fig. 4. Average of Cyclomatic Complexity (CM) per session (left), average of intermediate grades per session (right). 

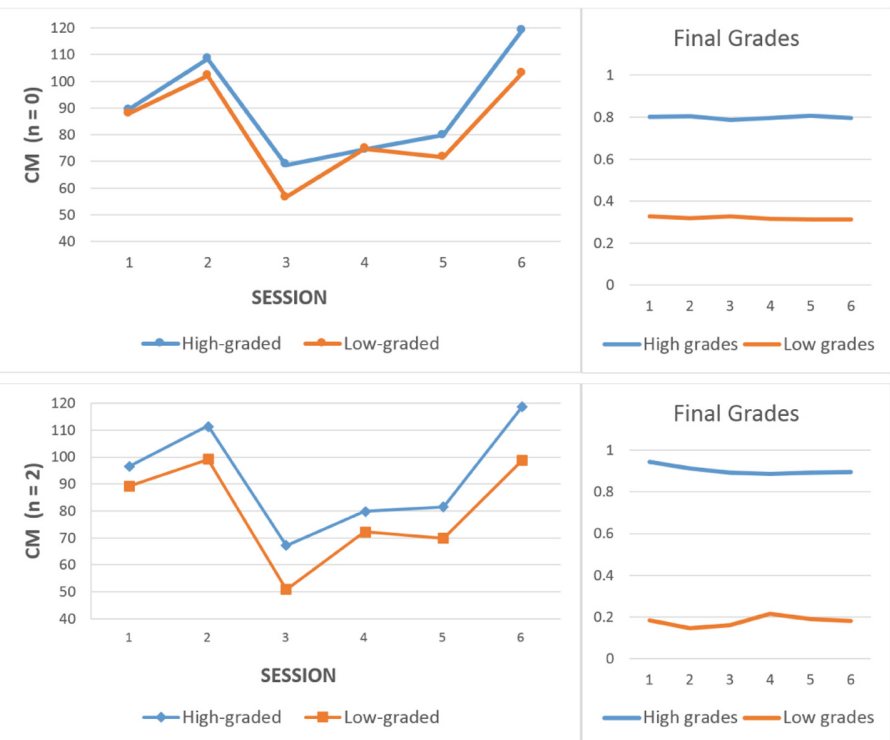

Fig. 5. Comparison of student clusters via the difference of average Cyclomatic Complexity $(\mathrm{CM})$ for the second level of granularity. The average final grades are shown on the right (the grades are normalized due to the privacy issues).
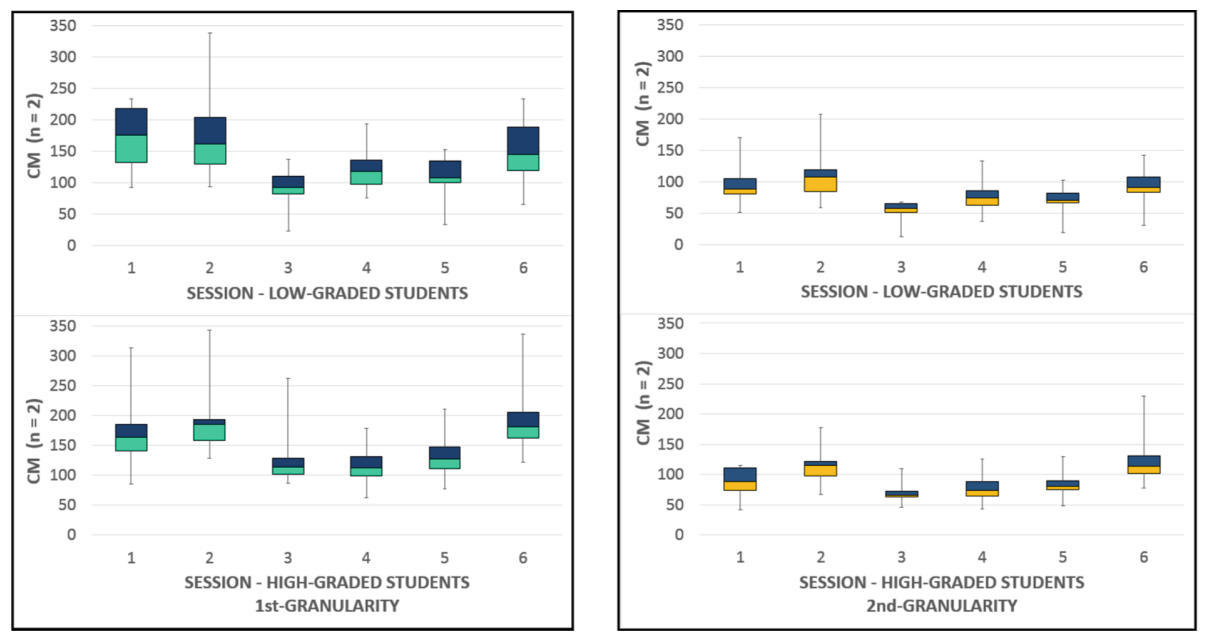

Fig. 6. The variance of average Cyclomatic Complexity (CM) for: Low-graded students (on the top) versus high-graded students (on the bottom), and first granularity level (on the left) versus second granularity level (on the right). 


\subsection{Impact of Granularity Level over Cyclomatic Complexity}

We also compared the variance of students $\mathrm{CM}$ between the most and the least successful students. For this comparison we considered the first and second level of granularity since we can compare their behavior via in-task versus out-oftask events. The results show that when considering all the events (Fig. 6 on the left), the behavior of high-graded students are closer to each other than the low-graded ones for the majority of sessions. Whereas, when we exclude the outof-task events in the second level (Fig. 6 on the right), the result is the opposite. The behavior of the least successful ones is closer to one another. And in both levels, this difference is higher in the sessions with more complex assignments. Additionally, a comparison of the two levels of granularity (Fig. 6) shows that the low-graded students perform more out-of-task activities that the better-graded students while interacting with Deeds.

\subsection{Instructors Insight}

The results obtained from a semi-structured interview of the instructors of the digital design course, are valuable for the interpretation of our findings. The interview questions ask them about: how difficult each course session was for the students, and how their experience was during the laboratory sessions. They were asked to sort the sessions based on the difficulty level from very difficult to very easy (Fig. 7). Also, the results of this study were shown to the instructors in order to get their interpretation and feedback.

Four instructors of the course were interviewed, each from 30 minutes to 1 hour. The instructors do not have the same level of experience. The major professor has over ten years experience of teaching with this particular course, and the experience of the rest varies from several years to several months. The results show that all agree on which sessions were the most difficult for the students (sessions 6 and 5) but their opinions varied about the difficulty level of

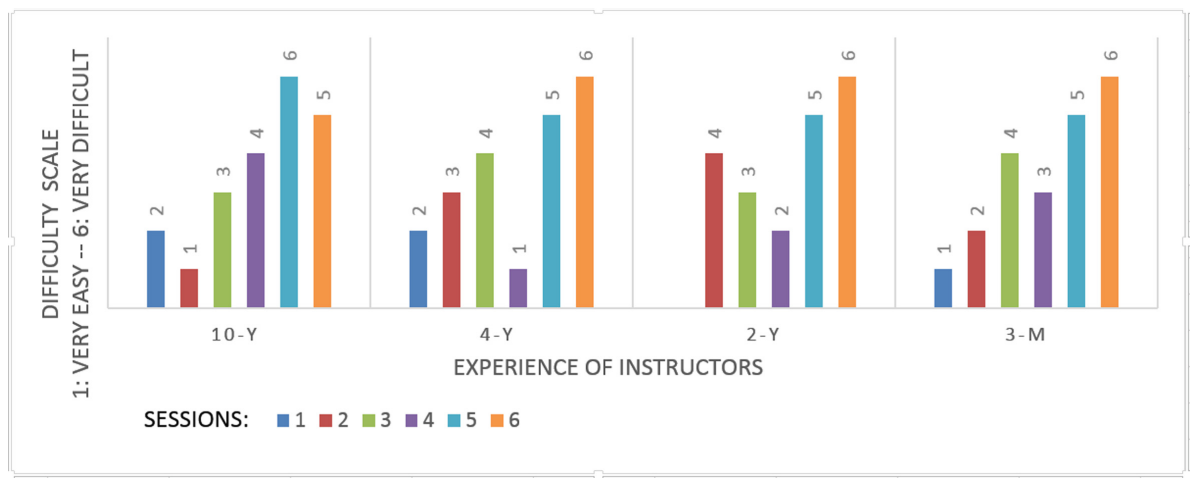

Fig. 7. The difficulty of sessions based on the instructors experience (from 10 years to 3 months). 
the rest of the sessions (see Fig. 7). For example, two teachers indicated session 4 as medium difficult and the two other indicated it as an easy session.

Also we asked the instructors to draw an expected process model of a student, given the main events. We compared the models drawn by instructors and observed that their process models were different in size of events and linear paths. Also, their opinions varied about the significance of relationship between various events. For instance, two indicated that increasing of linear paths is an indicator of less successful behavior and the other two stated that it is not an indicator of learning. These results confirm that the average $\mathrm{CM}$ of students and the obtained process models can give valuable insights to the instructors of the course, in particular to those with less experience.

\section{Discussion}

LA and EDM methods aim to improve our understanding about the behavior of learners while interacting with TEL systems. Researchers of the field attempt to adapt the analytics methods to the educational goals and contexts in order to gain better insight about the learning behavior of students. In this regard, applying emerging methods like PM is valuable to abstract the learning processes, leads to better detection of problems, and improves the learning outcome.

In this paper, we applied PM methods and metrics to understand and compare the learning processes of students. We obtained the data from interactions of students while they were working with an educational simulator named Deeds during six sessions of a course of digital design. A data-set of temporal and sequential activities per student and per course session is aggregated by LADC developed for our research. We applied complexity metric of CM as an understandability metric of a process model in order to compare the processes of student learning. We compared average CM of various clusters of students based on their academic achievements, and interviewed the four instructors of the course for their interpretation and feedback.

Our findings show that process discovery and CM of the student models can give insight about the learning process of students, and give valuable information about how students act in a process of problem solving. It was shown that fuzzy miner is an effective algorithm of PM to abstract and simplify the process models, to detect the irrelevant activities to the learning process, and to define the granularity levels. Additionally we found that: CM is positively correlated with the average final grades of students and inversely correlated with the difficulty level of laboratory sessions. As a result, average CM of students can be used as a predictor of the difficulty level of laboratory sessions for a group of students (from various engineering majors or intakes), and help instructors organize and adapt their lessons based on the needs of every group. We discovered that the successful students have more similar CM than the less successful ones, when considering all the activities including both in-task and out-of-task. On the contrary, the low-graded students have more similar CM when we exclude out-of-task activities. We propose the following hypothesis to explain this result: 
the better-graded students have more effortful cognitive activities and use more varied learning strategies in the process of problem solving.

In the future, we aim to use CM to predict the academic achievement of a group of students, and if possible, to predict for a single student. This can be done by adding other factors that influence the student success such as performance time, order of activities, etc. Such predictor can help teachers detect the students who deviate from the appropriate learning path so to provide help on time. Additionally, it can raise awareness of teachers to balance the difficulty of exercises based on the needs of the students with different academic backgrounds.

Acknowledgments. This work was supported in part by the Erasmus Mundus Joint Doctorate in Interactive and Cognitive Environments, which is funded by the EACEA Agency of the European Commission under EMJD ICE FPA n 2010-0012. Also, we thank professors Domenico Ponta and Giuliano Donzellini for providing support and help to our experiment.

\section{References}

1. Siemens, G.: Learning analytics: envisioning a research discipline and a domain of practice. In: International Conference on Learning Analytics and Knowledge (2012)

2. Chatti, M.A., Dyckhoff, A.L., Schroeder, U., Thüs, H.: A reference model for learning analytics. Int. J. Technol. Enhanced Learn. 4(5), 318-331 (2012)

3. Romero, C., Ventura, S.: Educational data mining: a survey from 1995 to 2005. Expert Syst. Appl. 33(1), 135-146 (2007)

4. Siemens, G., Long, P.: Penetrating the fog: analytics in learning and education. EDUCAUSE Rev. 46(5), 30 (2011)

5. Vahdat, M., Ghio, A., Oneto, L., Anguita, D., Funk, M., Rauterberg, M.: Advances in learning analytics and educational data mining. In: European Symposium on Artificial Neural Networks, Computational Intelligence and Machine Learning (2015)

6. Kruse, A., Pongsajapan, R.: Student-centered learning analytics. In: CNDLS Thought Papers (2012)

7. Prince, M.J., Felder, R.M.: Inductive teaching and learning methods: Definitions, comparisons, and research bases. J. Eng. Educ. 95(2), 123-138 (2006)

8. Vahdat, M., Oneto, L., Ghio, A., Anguita, D., Funk, M., Rauterberg, M.: Human algorithmic stability and human rademacher complexity. In: European Symposium on Artificial Neural Networks, Computational Intelligence and Machine Learning (2015)

9. Lee, V.S.: The power of inquiry as a way of learning. Innovative High. Educ. 36(3), 149-160 (2011)

10. De Jong, T., Sotiriou, S., Gillet, D.: Innovations in stem education: the go-lab federation of online labs. Smart Learn. Environ. 1(1), 1-16 (2014)

11. Donzellini, G., Ponta, D.: A simulation environment for e-learning in digital design. IEEE Trans. Ind. Electron. 54(6), 3078-3085 (2007)

12. Bienkowski, M., Feng, M., Means, B.: Enhancing teaching and learning through educational data mining and learning analytics: An issue brief. In: US Department of Education, Office of Educational Technology (2012) 
13. Del Blanco, A., Serrano, A., Freire, M., Martínez-Ortiz, I., Fernández-Manjón, B.: E-learning standards and learning analytics. can data collection be improved by using standard data models? In: IEEEGlobal Engineering Education Conference (2013)

14. Trčka, N., Pechenizkiy, M., van der Aalst, W.M.P.: Process mining from educational data. In: Robero, C., Ventura, S., Penchenizkiy, M., Baker, R., (eds.) Handbook of Educational Data Mining, pp. 123-142. Boca Raton: Chapman \& Hall/CRC (2010). http://www.crcnetbase.com/doi/abs/10.1201/b10274-11

15. Trcka, N., Pechenizkiy, M.: From local patterns to global models: Towards domain driven educational process mining. In: International Conference on Intelligent Systems Design and Applications (2009)

16. Pechenizkiy, M., Trcka, N., Vasilyeva, E., Van Der Aalst, W., De Bra, P.: Process mining online assessment data. In: International Working Group on Educational Data Mining (2009)

17. Bannert, M., Reimann, P., Sonnenberg, C.: Process mining techniques for analysing patterns and strategies in students self-regulated learning. Metacognition Learn. $\mathbf{9}(2), 161-185$ (2014)

18. Figl, K., Laue, R.: Cognitive complexity in business process modeling. In: Mouratidis, H., Rolland, C. (eds.) CAiSE 2011. LNCS, vol. 6741, pp. 452-466. Springer, Heidelberg (2011)

19. Gruhn, V., Laue, R.: Complexity metrics for business process models. In: International Conference on Business Information Systems (2006)

20. Štuikys, V., Damaševičius, R.: Complexity evaluation of feature models and metaprograms. In: Meta-Programming and Model-Driven Meta-Program Development (2013)

21. Lassen, K.B., Van Der Aalst, W.M.P.: Complexity metrics for workflow nets. Inf. Softw. Technol. 51(3), 610-626 (2009)

22. McCabe, T.J.: A complexity measure. IEEE Trans. Softw. Eng. 4, 308-320 (1976)

23. Zuse, H.: Softw. Complex. Walter de Cruyter, NY, USA (1991)

24. Thomas, J.C., Richards, J.T.: Achieving psychological simplicity: measures and methods to reduce cognitive complexity. In: Human-Computer Interaction: Design Issues, Solutions, and Applications (2009)

25. Rauterberg, M.: A method of a quantitative measurement of cognitive complexity. In: Human-Computer Interaction: Tasks and Organisation (1992)

26. Holzinger, A., Popova, E., Peischl, B., Ziefle, M.: On complexity reduction of user interfaces for safety-critical systems. In: Quirchmayr, G., Basl, J., You, I., Xu, L., Weippl, E. (eds.) CD-ARES 2012. LNCS, vol. 7465, pp. 108-122. Springer, Heidelberg (2012)

27. Ham, D.H., Park, J., Jung, W.: A framework-based approach to identifying and organizing the complexity factors of human-system interaction. IEEE Syst. J. 5(2), 213-222 (2011)

28. Rauterberg, M.: How to measure cognitive complexity in human-computer interaction. In: Cybernetics and Systems Research (1996)

29. Sapounidis, T., Demetriadis, S., Stamelos, I.: Evaluating children performance with graphical and tangible robot programming tools. Pers. Ubiquit. Comput. 19(1), 225-237 (2015)

30. Mohamed, N., Sulaiman, R.F., Endut, W.R.: The use of cyclomatic complexity metrics in programming performance's assessment. Procedia-Soc. Beha. Sci. 90, 497-503 (2013) 
31. Ponta, D., Anguita, D., Da Bormida, G., Donzellini, G.: Ten years of activity on computer-aided learning for electronics: Needs, experiences, field evaluation. In: Congreso sobre tecnologías aplicadas a la enseñanza de la electrónica (1998)

32. Vahdat, M., Oneto, L., Ghio, A., Donzellini, G., Anguita, D., Funk, M., Rauterberg, M.: A learning analytics methodology to profile students behavior and explore interactions with a digital electronics simulator. In: de Freitas, S., Rensing, C., Ley, T., Muñoz-Merino, P.J. (eds.) EC-TEL 2014. LNCS, vol. 8719, pp. 596-597. Springer, Heidelberg (2014)

33. Van Der Aalst, W.M.: Tool support. In: Process Mining (2011)

34. Günther, C.W., van der Aalst, W.M.P.: Fuzzy mining - adaptive process simplification based on multi-perspective metrics. In: Alonso, G., Dadam, P., Rosemann, M. (eds.) BPM 2007. LNCS, vol. 4714, pp. 328-343. Springer, Heidelberg (2007)

35. Van der Aalst, W., Weijters, T., Maruster, L.: Workflow mining: Discovering process models from event logs. IEEE Trans. Knowl. Data Eng. 16(9), 1128-1142 (2004) 